\title{
A Prediction Model for Hydrogen Induced Cracking in a Prestressed Wire with a Fracture Analysis
}

\author{
Taejun $\mathrm{CHO}^{1)}$ and TaeSoo $\mathrm{KIM}^{21}$ \\ 1) KR Technical Research Institute, Korea Rail Network Authority, Daejeon-si, 301-722, Korea. \\ 2) Division of Architectural Engineering, Hanbat National University, Daejeon, 305-719, Korea. E-mail: tskim0709@hotmail.com
}

(Received on October 1, 2007; accepted on December 17, 2007)

\begin{abstract}
This paper deals with the Hydrogen Induced Cracking $(\mathrm{HIC})$ behavior of high strength tendon wires of a prestressed structure, exposed to aggressive environments. A decoupling technique has been suggested to evaluate crack propagations in a wire section, driven by the hydrogen diffusion. By the proposed decoupling technique, three dimensional crack propagations can be modeled effectively by two 2-dimensional finite element models, one for the fracture analysis of a round bar wire in a longitudinal section, and the other for a hydrogen diffusion model in a horizontal section. Elastic and bi-linear elastic-plastic finite element analyses have been carried out for the evaluation of crack propagations in wires of tendon. A finite element program is developed for the hydrogen diffusion-stress analyses of wires. The decoupled analysis results have been compared with available test results, and showed improved results than the linearly assumed analytical equations suggested by Forman et al.
\end{abstract}

KEY WORDS: hydrogen induced cracking; decoupled analysis; crack propagation; high strength steel; prestressed structures

\section{Introduction}

\subsection{Backgrounds}

There are several reports on the structural failures by significant corrosion and Environmentally Assisted Cracking (referred to as EAC, hereafter) in high strength steel wire used for prestressed structures. ${ }^{1)}$ The cold drawn high strength steel wires used for prestressed structures are susceptible to corrosion, ${ }^{2)} \mathrm{EAC}$ and the resultant brittle failure. Although there were failures due to $\mathrm{EAC}$ in the tendons of prestressed structures, there has been little information about the details and analytical models. For the identification and prediction of safety of corroded infrastructures, an analytical model for EAC is strongly required.

The overall environmental effects on crack growth in high strength steel can be termed as Environmentally Assisted Cracking. EAC may include Stress Corrosion Cracking (referred to as SCC, hereafter), Hydrogen Assisted Cracking (referred to as HAC, hereafter), Low Cycle Fatigue, and Hydrogen Embrittlement of high strength steel. Except a Hydrogen Embrittlement, the phenomena are generally categorized by their strain rates. ${ }^{3)}$

When 3 conditions are met, SCC will take place specifically in certain metals in certain solutions (Fig. 1). It can happen under carbonated and bicarbonated, caustic, nitrate, mixture of nitric and sulfuric acid, mixture of magnesium chloride and sodium fluoride, hydrogen attack, cyanide, anhydrous ammnonia (liquid), or $\mathrm{CO} / \mathrm{CO}_{2} / \mathrm{H}_{2} \mathrm{O}$ mixtures. There have been a number of proposed mechanisms for the phenomenon, which can be classified either as an anodic
SCC mechanism or a mechanical fracture process. Anodic SCC involves the rupture of the protective oxide layer at the crack tip, anodic dissolution of the base metal, and crack growth under constant stress. Crack growth can be either intergranular or transgranular. ${ }^{3)}$

The other similar concept is that a film forms at the surface of a metal, and brittle fracture is induced by dealloying and/or vacancy injection. The crack proceeds through the film and across the film/metal interface into the metal where it propagates under the stress of the applied load. Once the crack stops, the process starts over with the for-

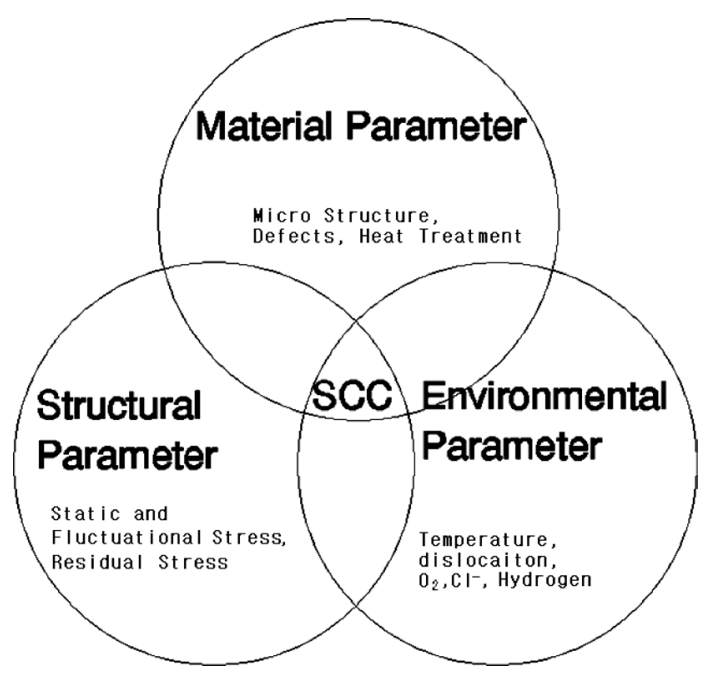

Fig. 1. SCC and parameters. 
mation of the film. It is notable that this mechanism is a combination of HAC and SCC.

Corrosion reactions often result in the formation of hydrogen gas. Hydrogen atoms can diffuse or are absorbed into the lattice of the metal causing a deterioration of the metal's properties. This, combined with the applied stress to the metal, can result in crack propagation. ${ }^{3)}$ Diffused hydrogen atoms can recombine to form hydrogen molecules, which can exert pressure on the surrounding steel, resulting in crack propagation in wire which is under high tensile stress. ${ }^{4)}$

The differences between SCC and HAC have been investigated by Wen-Ta Tsai and et al. ${ }^{5)}$ Depending on the potential, the forms and causes for the SCC $v s$. HAC were different. In their test, when the potential was moved toward the cathodic direction $(<-900 \mathrm{mV}$ SCE), HAC was observed. The loss of ductility as indicated by a decrease in the reduction of area of the specimen, compared with that in air test, was associated with the commencement and propagation of crack.

In all, hydrogen plays a key role in a number of cracking mechanisms, including SCC and HAC. HAC encompasses a number of different mechanisms, and in some cases is considered to be interchangeable with the phrases hydrogen-induced cracking (referred to as HIC, hereafter), hydrogen embrittlement, and hydrogen damage.

The mechanism of HAC involves the diffusion and buildup of gaseous hydrogen $\left(\mathrm{H}_{2}\right)$ in a void region of the metal. This process can easily occur if the structure is exposed to a hydrogen-rich environment. This gaseous hydrogen exerts a pressure against the walls of the metal, thus assisting in propagating the crack, or initiating a crack in the case where one doesn't already exist. The latter process can occur when $\mathrm{H}_{2}$ gas is "pushing" against the metal from the inside and augments the stress load being applied to the material so that the total stress exceeds that required to initiate a crack. This effectively creates an area of stress concentration and a nucleating site for a crack. The propagation of a crack is also assisted with the pressure of the hydrogen gas pushing against the crack walls from the inside, which again works in combination with the stress applied to the metal. Among the mechanisms of HAC, the cracking of a ductile alloy when under constant stress and where hydrogen gas is present could be defined as HIC. Hydrogen is absorbed into areas of high triaxial stress ultimately initiating the damage in high strength steel.

Focusing on the importance of hydrogen absorption to high strength steel which resulting crack propagation and brittle failure, this study has been concentrated on the development of an analytical model to identify the driving force and mechanism of HIC.

With regard to hydrogen diffusion-stress coupled analyses, numerous experimental researches have been carried out. Leeuwen ${ }^{6}$ and Yokobori ${ }^{7)}$ presented diffusion solutions by solving partial differential equations, respectively. Krom $^{4}$ investigated hydrogen gas pressure and hydrogen concentration relationships, and used the relation equations as the criteria of crack propagation in the elastic-plastic necking analysis of prestressing tendons, driven by hydrogen diffusion. The necking problem can be solved as axisymmetric, but the thumb-nail shaped cracks by hydrogen induced cracking in wires cannot. Based on the fracture toughness of hydride, Lufrano et al. ${ }^{8)}$ suggested a threshold of stress intensity factor for each crack propagation step.

Toribio calculated hydrogen embrittlement in the presence of residual stress fields. ${ }^{9)}$ The formulation of the model is based on stress-assisted hydrogen diffusion from the external environment in the metallic material and on a fracture criterion, including both the stress level and critical hydrogen concentration at the fracture point. He ignored crack propagations, but analyzed only the diffusion of hydrogen atoms. In contrast, hydrogen assisted cracking with crack propagation has been explained by many researchers, such as Leeuwen, $\left.{ }^{6}\right)$ Lufrano et al., ${ }^{8)}$ Toribio ${ }^{9)}$ and Krom et $a l^{4)}$ Krom and Lufrano considered by employing equilibrium conditions for the concentration of hydrogen between lattice and trap sites. The hydrogen concentration in trap sites is fairly independent of the strain rate due to the high binding energy. It is then quite reasonable to assume that we must take into account not the total amount of hydrogen but only the hydrogen in lattice sites.

To estimate the fracture toughness, Mayrbaurl et al. ${ }^{10)}$ tested 19 cracked wires taken during the inspection of a suspension bridge. For the purpose, tests of 19 wires for the hydrogen diffusion and stress-corrosion cracking in cable wires of a suspension bridge were conducted. The experimental results were compared with an analytical equation proposed by Forman et al. ${ }^{11)}$ In the tests, ${ }^{10)}$ the cracking led to sudden brittle failure when the crack depth was such that the stress intensity factor at the crack tip was equal to the critical stress intensity factor, fracture toughness $\left(K_{\mathrm{c}}\right)$ of the material. More details are discussed in Chap. 3.

\subsection{Objectives}

The main objective of this study is focused on the identification of the initiation and propagation of SCC and the resulting crack growth in prestressed tendon wires. For the purpose, the following research objectives are studied.

1) Decoupling modeling for the hydrogen induced cracking in a prestressed steel wire.

2) Development of an analytical model for the fracture analysis in a prestressed wire, with validation for the calculated results compared with the available test results.

3) Development of an analytical model for the diffusion analysis by the infiltrated hydrogen atoms, which uses the results of decoupled fracture analysis.

\section{Fracture Analysis Model}

\subsection{Decoupling the Diffusion Analysis and Fracture Analysis of SCC}

As can be seen in Fig. 2, a rigorous model may need a 3D full modeling of hydrogen diffusion analysis coupled with a fracture analysis. However, the 3D nonlinear analysis is so expensive that it often discourages to be selected in iterative design stages. In addition, the crack at the center surface showed the fastest crack propagation in fatigue crack growing tests ${ }^{12,13)}$ shown in Fig. 3. Accordingly for the sake of simplicity, the calculation of crack growing at the center of horizontal section is assumed as providing a conservative evaluation, which would contribute to the 


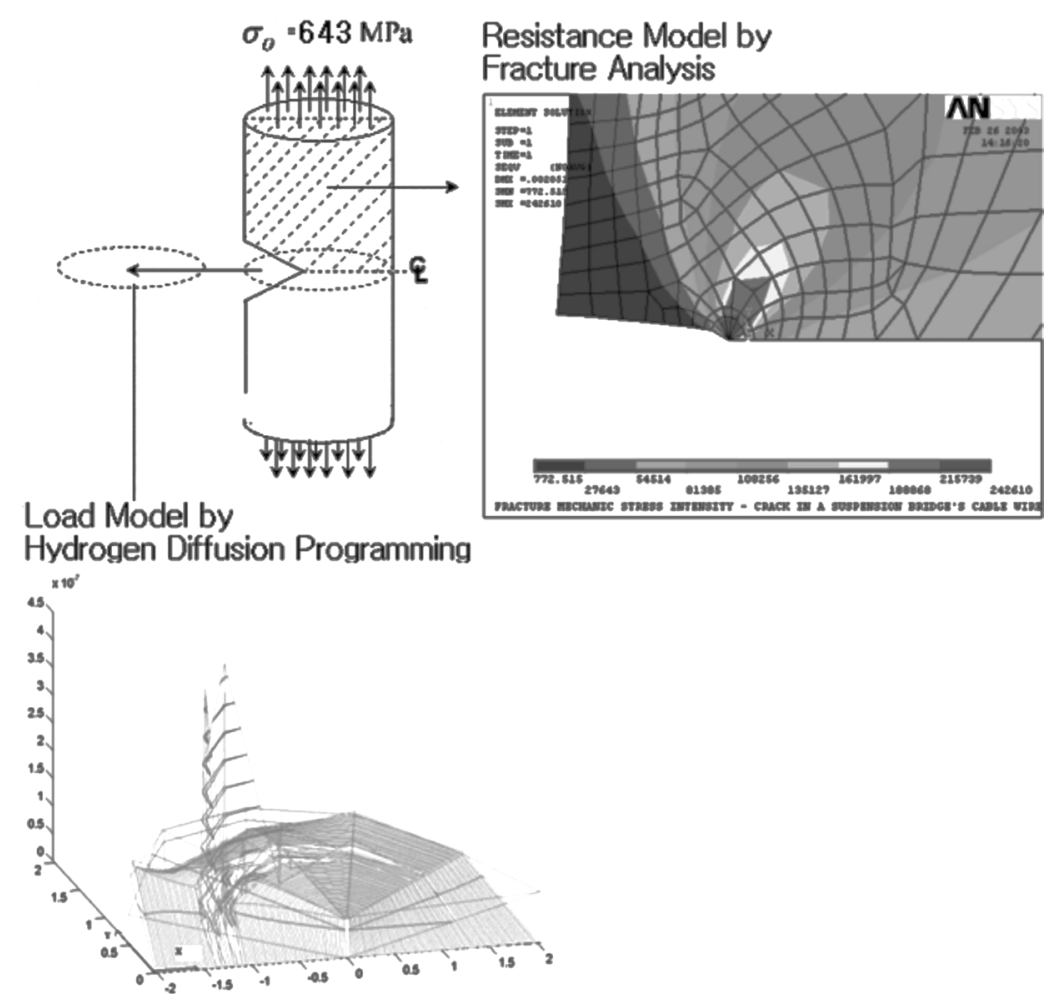

Fig. 2. Longitudinal and cross $2 \mathrm{D}$ sections of a tendon wire under tensile stress of $643 \mathrm{MPa}$, for the calculation of fracture analysis and diffusion analyzing programming, respectively.

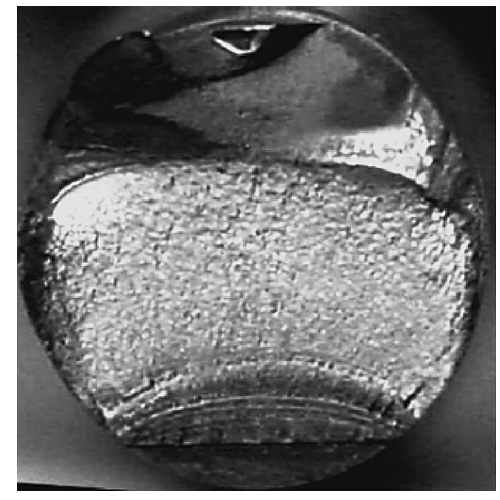

Fig. 3. Propagated fatigue crack shape on a round bar. ${ }^{13)}$

faster decision making during the iterative design of complicate structures.

Therefore, for the conservative but practical design of a prestressed structure regarding corrosion-deterioration, two 2 dimensional simplified finite element analysis models are proposed. In the longitudinal section the stress intensity factor is calculated by a fracture analysis using FE analysis program, and in the horizontal section, diffusion analysis with symmetric boundary condition has been programmed, which are compared with analytical and experimental results, respectively. In the proposed model, the external infiltration of hydrogen atoms is treated as load, which could be resisted by the strength of steel.

The suggested decoupling method provides various advantages over the analysis of coupled problems, such as diffusion-stress analysis. Pre-calculated stress intensity factors on each crack propagation step from the fracture analysis, significantly reduces the computational time. Furthermore, in more complex situations such as a sub-structural analy- sis, dynamic analysis, or other coupled analysis, the decoupling and parallel calculations generally show more stable results in more complex analyses including sub-structural analysis, dynamic analysis, etc. This is precisely the reason why the decoupling analyses of diffusion, stress, and fracture are dealt with separately in this paper. The stress intensity factor for the prestressed structural system has been calculated using a finite element analysis program, utilizing its elastic and bi-linear elastic-plastic material behavior. The acquired and assumed parameters and the results of the calculations are discussed in the following sections.

\subsection{Thumb-nail Shaped Crack Model}

\subsubsection{Geometry and Model}

Numerous researchers have studied the stress assisted hydrogen diffusion model. Toribio ${ }^{9)}$ analyzed hydrogen diffusion in a prestressed tendon by FEM methods, but he assumed the fracture of the tendon ignoring crack propagations. $\mathrm{Krom}^{4)}$ conducted a elastic-plastic necking analysis of prestressing tendons driven by hydrogen diffusion, and compared it with experimental results. The necking problem can be solved as axi-symmetric, but the thumb-nail shaped cracks from hydrogen induced cracking in wires can not. Leeuwen ${ }^{6}$ and Yokobori ${ }^{7)}$ published a diffusion solution by solving partial differential equations, respectively. Previous studies of crack propagation criteria are discussed in Secs. 3.1 and 3.2.

Figure 4 depicts the diffusion of hydrogen atoms with crack propagations with initial conditions of surface concentration of hydrogen atoms, and shape assumptions for the surface crack in a solid round bar, a tendon wire. As shown in Fig 4(a), the flux of hydrogen atom $(H)$ is modeled as the surface concentration. The dashed line in Fig. 4(b) shows the plane, on which the assumed crack flanks 


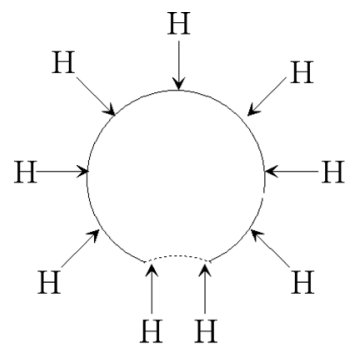

(a)

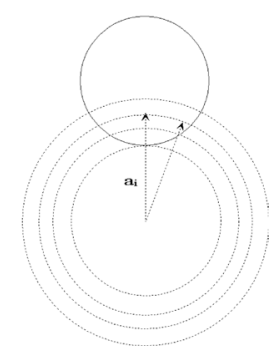

(b)
Fig. 4. Diffusion of hydrogen (a) initial condition, (b) assumed crack growth.

and crack tip are located. A plane strain hypothesis can be adopted, following a well known linear elastic solution in the vicinity of a 3D crack with an arbitrary curved front. ${ }^{9,14,15)}$ The assumed 2D model has the fixed circle at the center of crack growing (dashed lines with the radius of crack length as $a_{\mathrm{i}}$ ), and another circle for the tendon wire (solid line). The difference in the radii of the two crack propagations is determined by average stress intensity factor, and the estimation of the plastic zone size using the well known Irwin correction factor, $r_{y}$, which was also proposed in the previous study by Lin et al. ${ }^{16)}$

\subsubsection{Crack Tip Elements and Singular Quadratic Isoparametric Elements}

Since the crack tip causes a stress concentration, the stress and strain gradients are getting larger as the crack tip is approached. The difficulty in modeling Linear Elastic Fracture Mechanical (referred to as LEFEM, hereafter) problems using finite element method is that polynomial basis functions used for most conventional elements cannot represent the singular crack-tip stress and strain fields predicted analytically. Path dependence in the evaluation of the integral may be an indication that the mesh is not sufficiently refined. However, path independence does not prove mesh convergence. The finite element mesh must be refined in the vicinity of the crack to get accurate stresses and strains; however, accurate $J$-integral results can frequently be obtained even with a relatively coarser mesh.

The problem of finite element method for LEFM was significantly reduced by Barsom. ${ }^{17)} \mathrm{He}$ showed that the proper crack-tip displacement, stress, and strain fields are modeled by standard quadratic order isoparametric finite elements. The broken 8-noded isoparametric element for plane strain analysis is achieved by placing the mid-side node near the crack tip at the quarter point. Such elements in the non-singular formulation satisfy the essential convergence criteria. This procedure introduces a singularity into the mapping between the element's parametric coordinate space and Cartesian space ${ }^{17)}$ where the crack-tip nodes $(1,4,8)$ are constrained to move together.

The sizes of the crack-tip elements influence the accuracy of the solutions: the smaller the radial dimension of the elements, the better the results will be. The angular strain dependence is not modeled with the singular elements. Reasonable results are obtained if typical elements around the crack tip subtend angles in the range of $10^{\circ}$ (accurate) to $22.5^{\circ}$ (moderately accurate). The triangle crack tip element is generated by collapsing side $1-4$ of the

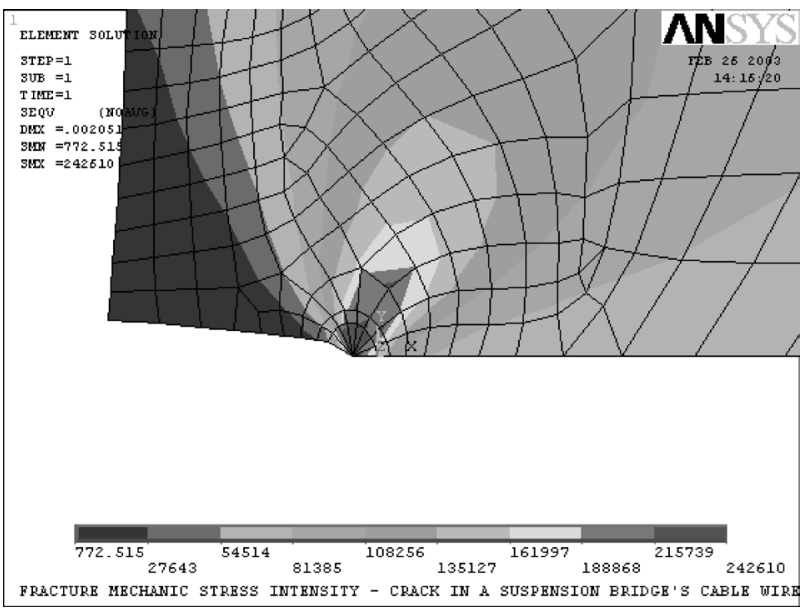

Fig. 5. Crack tip elements around crack tip area, under external pressures with crack growth.

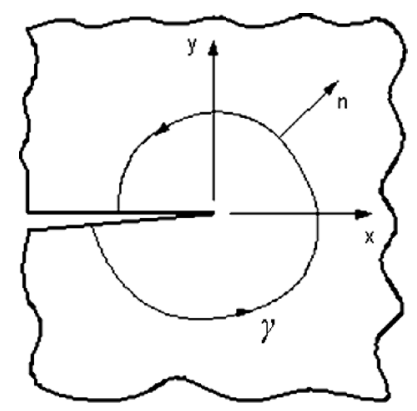

Fig. 6. $J$-integral contour path surrounding a crack-tip.

quadrilateral nodes. In this case, the singularity is investigated along the $x$-axis, $y=0$. An example figure of crack tip elements in the fracture analysis is shown in Fig. 5. The SIF at each crack propagating step is calculated by two methods. First one is using a definition of SIF as using stress and crack length calculated by FE analysis. The other one is path independent contour integral method, described in the next section.

\subsection{J-Integral}

The SIF for pure model I crack problems can be evaluated using a domain-form of the contour $J$-integral. The interaction integral formulation is used to extract the SIF, $K_{\mathrm{I}}$ as given by, ${ }^{18)}$

$$
K_{\mathrm{I}}=\sqrt{\frac{J \cdot E}{1-v^{2}}}
$$

where $K_{\mathrm{I}}$ is Mode I SIF, $J$ is a path-independent line integral, $v$ is the Poisson ratio.

As shown in Fig. 6, the $J$-integral can be defined as a path-independent line integral that measures the strength of singular stresses and strains near a crack tip (Eq. (2)). In 2$\mathrm{D}$ form, the following procedures of calculation were used. This calculation assumes that the crack lies in the global Cartesian $\mathrm{X}-\mathrm{Y}$ plane, with $\mathrm{X}$ parallel to the crack:

$$
J=\int_{\gamma} W d y-\int_{\gamma}\left(t_{x} \cdot \frac{\partial u_{x}}{\partial x}+t_{y} \cdot \frac{\partial u_{y}}{\partial x}\right) d s
$$

where $\gamma=$ any path surrounding the crack tip, $W=$ strain energy density (that is, strain energy per unit volume), 
$t_{x}=$ traction vector along $x$ axis $\left(=\sigma_{x} \cdot n_{x}+\sigma_{x y} \cdot n_{y}\right), t_{y}=$ traction vector along $y$ axis $\left(=\sigma_{y} \cdot n_{y}+\sigma_{x y} \cdot n_{x}\right), \sigma=$ component stress, $n=$ unit outer normal vector to path $\gamma, u=$ displacement vector, $s=$ distance along the path $\gamma$.

Following STEPs are implemented in a script file of ANSYS for the calculation of contour integral by Virtual Crack Extension Method to calculate the $J$ value:

STEP1. Read in the desired set of results of stress analyses.

STEP2. Store the volume and strain energy per element.

STEP3. Calculate the strain energy density per element.

STEP4. Define a path for the line integral.

STEP5. Map the strain energy density, which was stored in the element table in step 1, onto the path.

STEP6. Integrate it with respect to global Y.

STEP7. Assign the final value of the integral to a parameter.

STEP8. Map the component stresses, SX, SY, and SXY onto the path.

STEP9. Define the path unit normal vector.

STEP10. Calculate traction vectors, $t_{x}$ and $t_{y}$.

STEP11. Shift the path a small distance in the positive and negative $\mathrm{X}$ directions to calculate the derivatives of the displacement vector $\left(\delta_{u x} / \delta_{x}\right.$ and $\left.\delta_{u y} / \delta_{y}\right)$. The following steps are involved (see STEP12) Calculating derivatives of the displacement vector).

STEP13. Calculate the distance by which the path is to be shifted, say DX. A rule of thumb is to use one percent of the total length of the path.

STEP14. Shift the path a distance of DX/2 in the negative $\mathrm{X}$ direction and map the displacements, UX and UY, onto the path, giving them labels as UX1 and UY1, for example.

Shift the path a distance of DX in the positive $\mathrm{X}$ direction (that is, $+\mathrm{DX} / 2$ from its original position) and map UX and UY onto the path, giving them labels UX2 and UY2, for example.

Shift the path back to its original location (a distance of $-\mathrm{DX} / 2$ ) and calculate the quantities (UX2-UX1)/DX and (UY2-UY1)/DX. These quantities represent $\delta_{u x} / \delta_{x}$ and $\delta_{u v} / \delta_{y}$, respectively.

STEP15. Using the quantities calculated in steps 10 and 11 , calculate the integrand in the second term of $J$ and integrate it with respect to the path distance $S$.

STEP16. Calculate $J$-integrations, using the quantities calculated in STEPs 5-7 and 12.

The calculation steps are programmed in a macro file as commands for ANSYS, for $a / b=0.05$, prestress $=524 \mathrm{MPa}$, for comparison with test results discussed in the following section.

\subsection{Comparison with the Previous Test Results}

Thumb-nail shape hydrogen induced crackings on a tendon wire were found by Mayrbaurl et al.'s tests for HIC. ${ }^{10)}$ While crack growing, the diffused hydrogen atoms by the difference of tensile stress gradient are combined as molecules, which produce gas pressure and embrittle the high strength steel. In the 19 wire tests, the cracks lead to sudden brittle failure when the stress intensity factor at the crack tip is equal to the critical stress intensity factor, or the fracture toughness $\left(K_{\mathrm{c}}\right)$ of the material. The stress intensity fac- tor (SIF), $K_{\mathrm{I}}$ for combined axial and bending stress is obtained by adding the results from the two formulas, which is given by Forman et al. ${ }^{11)}$

The stress intensity factor $\left(K_{\mathrm{I}}\right)$ for an axially stressed solid cylindrical specimen with a thumb-nail shaped crack is given by Forman et al. ${ }^{11)}$ as follows:

$$
K_{\mathrm{I}}=s_{\mathrm{a}} \cdot \sqrt{\pi \cdot a} \cdot F(a / b)
$$

For a solid cylindrical specimen with an applied pure bending moment, the stress intensity factor is given by the Eq. (3), replacing the axial stress $\left(s_{\mathrm{a}}\right)$ with the bending stress $\left(s_{\mathrm{b}}\right)$ :

$$
K_{\mathrm{I}}=s_{\mathrm{b}} \cdot \sqrt{\pi \cdot a} \cdot F(a / b), \quad s_{\mathrm{b}}=\frac{32 M}{\pi b^{3}}
$$

where, $M$ is bending moment in the cylinder, and

$$
\begin{aligned}
& F(a / b)= 0.92 \cdot \frac{2}{\pi} \cdot \sqrt{\frac{2 b}{\pi a} \tan \frac{\pi a}{2 b}} \\
& \times \frac{0.752+1.286 \cdot(a / b)+0.37 \cdot\left(1-\sin \frac{\pi a}{2 b}\right)^{3}}{\cos \frac{\pi a}{2 b}} \\
& F(a / b)= 0.92 \cdot \frac{2}{\pi} \cdot \sqrt{\frac{2 b}{\pi a} \cdot \tan \frac{\pi a}{2 b}} \\
& \times \frac{0.923+0.199\left(1-\sin \frac{\pi a}{2 b}\right)^{4}}{\cos \frac{\pi \ldots}{2 b}} \ldots \ldots \ldots \ldots \ldots \ldots \ldots \ldots \ldots \ldots \ldots \ldots \ldots \ldots \ldots \\
&
\end{aligned}
$$

The stress intensity factor $\left(K_{\mathrm{I}}\right)$ for combined axial and bending stress is obtained by adding the results from the two formulas. As shown in Fig. 7, the stress intensity factor calculated for the stresses at the fracture of the test specimens defines the fracture toughness of the tendon wire. The values have been calculated using the tensile strength of

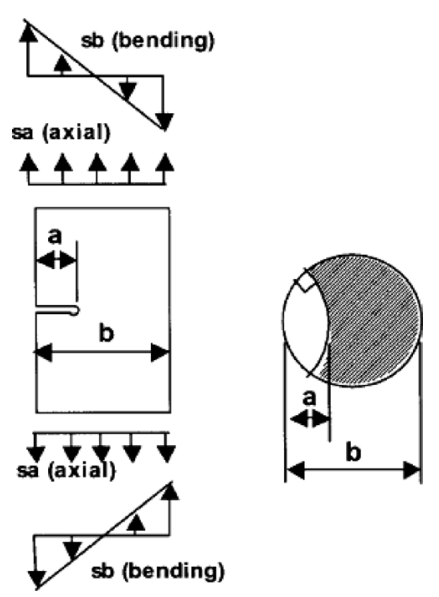

Fig. 7. Solid cylindrical rod with thumb-nail crack perpendicular to its axis, showing stress due to axial forces and uniform bending moment by Mayrbaurl et al. ${ }^{10)}$ 
each specimen, the estimated crack depth, and the bending stress of $524 \mathrm{MPa}$, that results from pulling the wire straight. Accordingly, the resultant SIF is the addition of axial and bending component, which is given by,

$$
K_{\mathrm{I}}=s_{\mathrm{a}} \cdot \sqrt{\pi \cdot a} \cdot F(a / b)+\frac{32 M}{\pi b^{3}} \cdot \sqrt{\pi \cdot a} \cdot F(a / b)
$$

As shown in Fig. 8, the mean value of the stress intensity factor, $62.2 \mathrm{MPa} \sqrt{\mathrm{m}}$, is very close value, compared with the result of the experiments. ${ }^{10)}$ However, there is a discrepancy; if considering the crack depth/diameter ratio by the equation of Forman et al., ${ }^{11)}$ where $K$ (Axial) and $K$ (Bending) are calculated by Eq. (3) and Eq. (4), respectively, while $K$ (Axial+Bending) is the sum of the two equations. Hence, the real stress and cracking, affected not only axial force but bending moment as well, is compared in the figure. However, it is also found that the linearly approximated prediction equations for SIF considering both axial and bending forces still show differences with the real behavior. Moreover, the equations (Eq. (3) and Eq. (4)) could not predict the fracture point in terms of a crack length in the section.

Employing bi-linear elastic-plastic material behavior and contour integral methods has reduced the differences. Combined with FE analysis the path independent contour integral method provides accurate computation of stress intensity factors for isotropic materials. At each crack propagation step, the SIF values were calculated by using a command of KCALC in a finite element program of ANSYS based on homogeneous isotropic linear elastic fracture me-

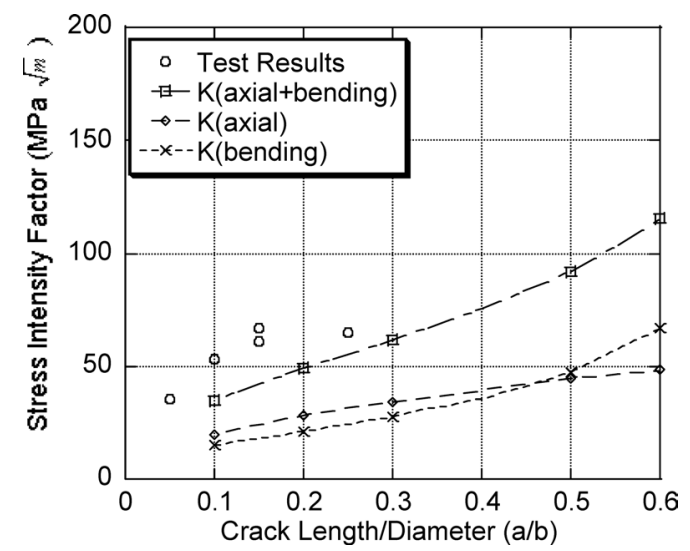

Fig. 8. Calculated SIFs by axial, bending, and combined external forces, compared with test results by Maryrbaurl. ${ }^{10)}$ chanics. The first row of plane elements (PLANE82, the 6node triangular solid) around the crack tip should be singular, which is modeled by utilizing the command of KSCON with the option of skewing midside nodes of the first row of elements to the $1 / 4$ point for crack tip singularity, ${ }^{17)}$ resulting the model of the crack tip elements.

As observed in Fig. 9 and Table 1, contour integral method is used for the evaluations of SIF values, which is in general well correlated with the experimental results, as providing the information of fracture points in terms of crack length over diameter $(a / b)$ in X-axis (blue rectangular line) based on the tensile fracture of the material, while the analytical equations ( $K$ (Axial+Bending $))$ and the linear elastic fracture method (green diamond line) could not predict the failure point.

In the bi-linear elastic-plastic material model (black cross line), the SIF values are calculated until they meet the limitation of tensile strain of steel as 0.164 . When the section fails by the material property of tensile failure, the calculated SIF also shows a rather close value with the test results of fracture toughness for the specimen, the mean value of SIF, $57.78 \mathrm{MPa}$. Until the section fails by the tensile elongation, the SIF calculated in the bi-linear elastic-plastic material model can be calculated to the depth ratio $(a / b)$ of 0.24 , at which the SIF reaches to the fracture toughness value $\left(K_{\mathrm{c}}\right)$. Hence, in the section considered, the fracture

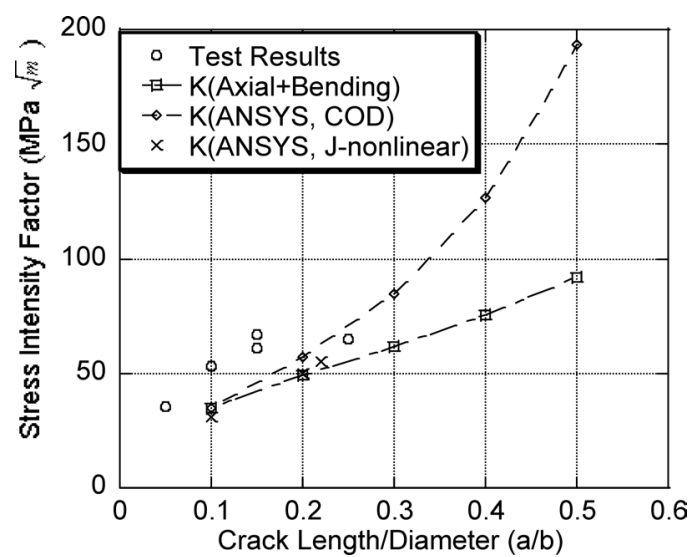

Fig. 9. Calculated SIF by elastic and bi-linear elastic-plastic material behavior, in this paper and test results by Maryrbaurl, ${ }^{10)}$ where $K$ (ANSYS, COD) and $K$ (ANSYS, $J$ integral) are calculated by using the definition of SIF and by using contour integral method, respectively, while $K$ (ax+bend) is the sum of the two equations (Eqs. (3) and (4)).

Table 1. Extracted data from the part of analysis, compared with test results.

\begin{tabular}{cccccccc}
\hline Index & $a / b$ & $\begin{array}{c}\text { Test } \\
\text { Results }\end{array}$ & $\begin{array}{c}\mathrm{K} \\
\text { (Axial) }\end{array}$ & $\begin{array}{c}\mathrm{K} \\
\text { (Bending) }\end{array}$ & $\begin{array}{c}\mathrm{K} \\
\text { (Axial+Bending) }\end{array}$ & $\begin{array}{c}\mathrm{K} \\
\text { (ANSYS, (ANSYS, J- } \\
\text { COD) }\end{array}$ & $\begin{array}{c}\mathrm{K} \\
\text { nonlinear) }\end{array}$ \\
\hline 1 & 0.1 & 51.22 & 19.81 & 14.98 & 34.79 & 34.76 & 31.09 \\
Difference & & $0 \%$ & & & $32.08 \%$ & $32.14 \%$ & $39.30 \%$ \\
2 & 0.2 & 60 & 28.02 & 21.08 & 49.1 & 56.89 & 50.06 \\
Difference & & $0 \%$ & & & $18.17 \%$ & $5.18 \%$ & $16.57 \%$ \\
3 & 0.22 & & & & & & 53.36 \\
Sum & & 982.18 & & & 41.95 & 45.83 & 45.5 \\
Mean & & 57.78 & & & $27.40 \%$ & $20.68 \%$ & $21.25 \%$ \\
Difference & & $0 \%$ & & & &
\end{tabular}


Table 2. Comparison of governing equation for stress assisted hydrogen diffusion.

\begin{tabular}{|c|c|c|c|}
\hline Authors & $\begin{array}{c}\text { Crack } \\
\text { Initiation }\end{array}$ & Propagation & Failure Criteria \\
\hline J. Toribo & Un-cracked & Un-cracked assumed & $c_{c}, x_{c}$ \\
\hline \multicolumn{4}{|c|}{ Hydrogen gas pressure, $\mathrm{z}$ and } \\
\hline A. Krom & Cracked & $\mathrm{LFM} ; K_{I}=\frac{2 P}{\pi} \sqrt{\pi a}>K_{C}$ & {$\left[K_{C}\right]_{\min }$ from test } \\
\hline T. Yokobori & $\begin{array}{l}\text { Diffusion } \\
\text { only }\end{array}$ & Diffusion only & $\begin{array}{c}\text { Critical concentration of } \\
\text { diffused hydrogen }\left(C_{c r}\right) \\
\text { as, } \\
C_{c r} / C_{o}=3.0\end{array}$ \\
\hline V. V. Bolotin & $\begin{array}{l}\text { Incubation } \\
\qquad \begin{array}{c}G<\Gamma \\
\text { Initiation } \\
G=\Gamma\end{array}\end{array}$ & $\begin{array}{l}\text { Result of interaction between the global } \\
\text { balance of forces and energy in the cracked } \\
\text { system }\end{array}$ & $\begin{array}{c}\text { Fracture toughness } \\
\text { calculated form specific } \\
\text { fracture work }\end{array}$ \\
\hline \multicolumn{4}{|c|}{$K_{I}=S_{a} \sqrt{\pi a} F(a / b)$} \\
\hline $\begin{array}{c}\text { R. } \\
\text { Maryrbaurl }\end{array}$ & $\begin{array}{l}\text { Breakage of } \\
\text { zinc coating } \\
\text { by general } \\
\text { corrosion }\end{array}$ & $\begin{array}{c}\text { for thumb nail crack [Forman(1994)] } \\
F(a / b)=0.92 \frac{2}{\pi} \sqrt{\frac{2 b}{\pi a} \tan \frac{\pi a}{2 b}} \frac{0.752+1.286(a / b)+0.37\left(1-\sin \frac{\pi}{2 l}\right.}{\cos \frac{\pi a}{2 b}}\end{array}$ & 19 wire test results \\
\hline
\end{tabular}

point could be predicted either by examining tensile strain or by examining stress intensity factor, compared with the fracture toughness.

In the linear elastic material model, however, because only one value of Modulus of Elasticity is used as $19700 \mathrm{MPa}$, the SIF values can be calculated until the depth ratio $(a / b)$ of 1.0. Therefore, the linear elastic model has only one criterion of fracture toughness to determine the fracture of the section.

While crack grows in Table 1, $K$ (Axial+Bending) shows the largest difference of $27.41 \%$ among analytical and $\mathrm{FE}$ analyses, compared with the mean value of test results. Consequently, the linearly assumed analytical equation underestimates the SIF values, even in the case of considering both axial forces and bending moments. It is further notable that the proposed finite element models rapidly narrow the difference with the test results as cracks grow from 10 to $22 \%$ of the diameter, while the Forman's analytical equation shows slowly decreasing differences.

In short, the analytical prediction of fracture point of a high strength steel section, affected by hydrogen induced cracking, is essential for the maintenance of a prestressed structure during the life span. In this comparative fracture analysis of a prestressed wire section, the proposed bi-linear elastic plastic finite element fracture analyses procedure provides an improved prediction results for the fracture points based on two criteria, tensile fracture and stress intensity factor, which could combined with the diffusion analysis to prevent a sudden failure of a prestressed structure, described in the subsequent sections.

\section{Diffusion Analysis Model}

\subsection{Governing Equation for the Diffusion of Hydrogen Atoms}

Hydrogen diffusion-stress coupled analyses and tests for stress-corrosion cracking have been studied many researchers. ${ }^{4,6-10)}$ As compared in Table 2, Yokobori used fi- nite difference methods for the time-dependent diffusion analysis, while Yokobori and Krom calculated the diffusion of hydrogen by finite element methods respectively.

For the time and crack growth-dependent finite element analysis for SCC, finite element formulation is adopted following the Krom's study. ${ }^{4)}$ However, the modeling of crack shape and coupled analysis are changed to model the thumb nail shaped crack on prestressed wires by the proposed decoupling method. Therefore, the formulation of the model is based on stress-assisted hydrogen diffusion from the external environment in the metallic material and on a fracture criterion, including both the stress level and critical hydrogen concentration at the fracture point.

\subsection{Finite Element Formulation for the Governing Equation of SCC}

A closed solution is not in general obtained from diffusion equations and thus numerical integration, such as finite difference method has been employed to solve such equations. Another widely accepted approach is a finite element method. In particular, the finite element method is of popular use in order to take into account the effects of convection, sorption, tensile stress, and etc.

Therefore, a transient stress assisted hydrogen diffusion analysis finite element program has been developed for the evaluation of hydrogen diffusion into the location of crack growth of high strength steel. The governing equations are an extension of Fick's equations, to allow for non-uniform solubility of the diffusing substance through the base material. The basic solution variable, for which the degrees of freedom are determined at each node, is the "hydrogen concentration".

Applying hydrogen mass conservation and divergence theorem, the governing equation is satisfied in weak sense for the hydrogen diffusion with the assumed essential boundary condition of prescribed hydrogen concentration. Diffusion is assumed to be driven by the gradient of general chemical potential. Hence, the hydrogen-diffusion equation 
for a body with volume $V$ and surface $S$ is then given by,

$$
\int_{V} \frac{\partial}{\partial t}\left(C_{\mathrm{L}}+C_{\mathrm{T}}\right) d V+\int_{S} \mathbf{n} \cdot \mathbf{J} d S=0
$$

where $\partial / \partial t$ is the partial derivative with respect to time, and $C_{\mathrm{L}}$ and $C_{\mathrm{T}}$ is the hydrogen concentration at lattice and trap sites each, $V$ is any volume whose surface is $S, \mathbf{n}$ is the outward-pointing unit normal vector, $\mathbf{n} \cdot \mathbf{J}$ stands for the concentration flux leaving $S$, with a volume $V$ boundary by a surface $S$, which consists of a part $S_{\mathrm{c}}$ where the hydrogen concentration is prescribed $C_{0}$, and a part $S_{\phi}$ where the flux through the surface is prescribed by a value $(\phi)$, and $\mathbf{J}$ is the flux of concentration of the diffusing phase.

The governing equation is derived using a divergence theorem and is expressed in a matrix form. A linear backward difference scheme is used for the discretization of the time variable. A simple estimate of the matrices $[M],[K]$, and $\{F\}$ at $t+\Delta t$ can be made, which is given by,

$$
\left([M]_{\mathrm{t}}+\Delta t \cdot\left[K_{1}\right]\right)\{C\}^{t+\Delta t}=\left([M]_{\mathrm{t}}-\Delta t \cdot\left[K_{2}\right]\right)\{C\}^{t}+\{F\}^{t}=0
$$

where $[M]=\int_{V}[N]^{\mathrm{T}} \cdot D \cdot[N] d V$,

$[N]=$ matrix of a shape function,

$$
\left[K_{1}\right]=\int_{V}[B]^{\mathrm{T}} \cdot D \cdot[B] d V,
$$

$[B]=$ derivative of matrix of a shape function,

$$
\begin{aligned}
& {\left[K_{2}\right]=-\int_{V}[B]^{\mathrm{T}} \cdot \frac{D_{\mathrm{L}} \cdot \overline{V_{\mathrm{H}}}}{R \cdot T} \cdot[B] \cdot\left[\sigma_{\mathrm{h}}\right] \cdot[N] d V,} \\
& \{F\}=\sum_{\text {elements }} \int_{S_{\phi}}[N]^{\mathrm{T}} \cdot \phi \cdot d S,
\end{aligned}
$$

$D_{\mathrm{L}}$ is a concentration independent lattice diffusivity, $V_{\mathrm{H}}$ is the partial molar volume of hydrogen, $R$ is the universal gas constant (i.e., $8.3144 \mathrm{~J} \mathrm{~mol} / \mathrm{K}), T$ is the absolute temperature, $\nabla \sigma_{\mathrm{h}}$ is the gradient of hydrostatic stress, and $\Delta t$ denotes the time increment. More details for a finite element formulation can be found in reference. ${ }^{4)}$

Using the above, a transient finite element analysis program has been developed for the calculation of hydrogen diffusion affected by the tensile stress gradient. The initiation of corrosion is calculated when the diffused chloride ion reaches to the defined threshold value. A detailed algorithm of the developed finite element analysis program with regard to the numerical time integration can be found elsewhere. ${ }^{19,20)}$

The authors developed a finite element code for the resultant differential equation (Eq. (9)) by using an equivalent pressure around the crack tip, pre-calculated in the fracture analysis described in Chap. 2. Accordingly, the hydrostatic stress at the nodes, $p$, is determined, which is given by,

$$
p=-\frac{K \cdot(1+v)}{\sqrt{2 \cdot \pi \cdot r}}-\frac{(1+v) \cdot \sigma}{3} .
$$

where $K$ is the stress intensity factor for a Mode I crack of length $r, v$ is poisson's ratio and $\sigma$ is the externally applied distributed load. With the use of the analytical values of equivalent pressure stress, the concentration solution by the FE program will show identical results with limited analytical concentration cases.

\subsection{Flowchart of the Proposed Decoupled Analysis for the SCC in Prestressed Wires}

As aforementioned in Sec. 2.1, two 2 dimensional decoupled finite element analysis models are used for the hydrogen induced crack growing by the diffusion of hydrogen atoms. Therefore, in the longitudinal section the stress intensity factor is calculated by a fracture analysis using FE analysis program, and in the horizontal section diffusion analysis with symmetric boundary condition has been programmed. As shown in Fig. 10, the time-dependent diffusion analysis program uses the results of fracture analysis, among which hydrostatic stresses and crack opening displacement is used for the calculation of equivalent concentration of diffused hydrogen by applying Eq. (9).

The relationship among the calculated pressure from previous volume-pressure relations, the critical concentration of hydrogen at each step is determined based on the empirical observations. The equivalent concentration of diffused hydrogen atoms is calculated by utilizing gas pressure obtained by Eq. (9), which needs the pre-calculated hydrostatic stress and displacement information based on the fracture analysis. The volume is calculated using the displacements, which are obtained from the fracture analysis at each crack propagation step. The combined gas pressure $(p)$ of hydrogen, the volume of the hydrogen gas $(V)$, at the temperature $(T)$ while crack propagates, may be obtained from the collected experimental data and the formulation of $p-V-T^{5,6)}$ as:

$$
p=\frac{n \cdot R \cdot T}{\left(V-n \cdot R \cdot T \cdot B_{2}\right)}, \quad B_{2}=\frac{z_{1}}{T}+Z_{2}
$$

where $n$ is the number of hydrogen moles, $B_{2}$ is the second virial coefficients, $z_{1}$ and $z_{2}$ are $1.51 \mathrm{e}^{-6} \mathrm{~K} / \mathrm{Pa}$, and $-1.04 \mathrm{e}^{-11} 1 / \mathrm{Pa}$, respectively. ${ }^{4}$

Accordingly, the relationship between the concentration of hydrogen atoms and gas pressure is given by,

$$
C=k \cdot \sqrt{p \cdot \exp \left\{\left(\frac{z_{1}}{T}+z_{2}\right) \cdot p\right\}}
$$

where $k$ is solubility of hydrogen in iron $\left(=0.82 \mathrm{e}^{-28600 / R T}\right)$.

Crack grows with varying boundary conditions and elements, when the equivalent concentration of hydrogen reaches to the value needed for crack growing. The time dependent diffusion of hydrogen with crack propagation is continuously calculated at each crack steps until the SIF meet with fracture toughness of the steel. An application example of the developed SCC programs to a prestressed concrete structure is introduced in a separate paper. 


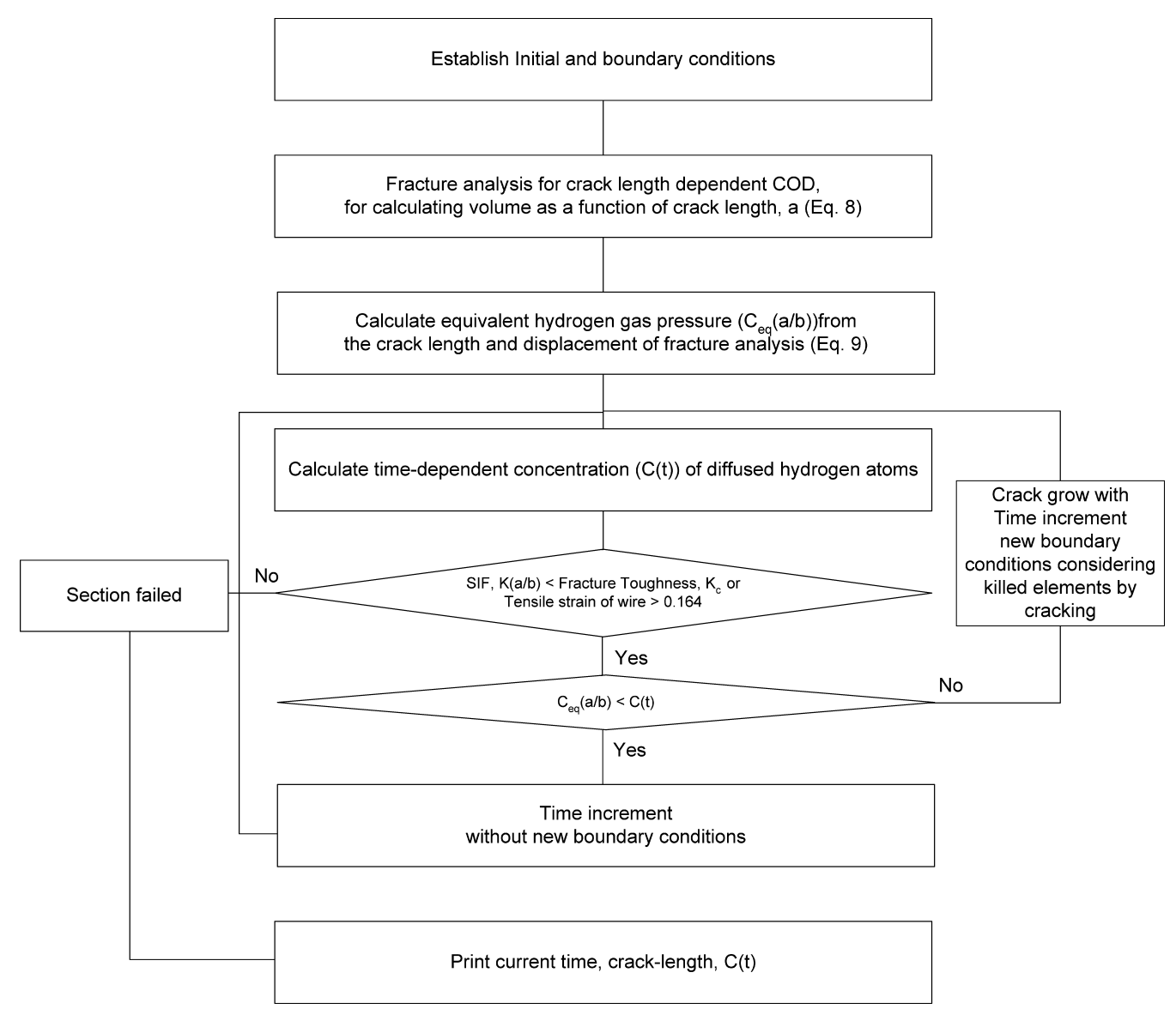

Fig. 10. Flowchart of the decoupled SCC analysis program.

\section{Conclusion Remarks}

A simplified analytical model for the crack length dependent hydrogen induced cracking in wires of tendon is proposed. The major findings are as follows:

(1) A decoupling technique is proposed for the evaluation of crack propagation in a wire section driven by the hydrogen diffusion. The decoupling technique incorporates a 3-dimensional crack propagation effectively by two 2-D finite element models: One for the fracture analysis of a round bar wire in the longitudinal direction, and the other for a hydrogen diffusion model in a transverse direction.

(2) In the fracture analysis for a longitudinal section of a prestressing wire, elastic and bi-linear elastic-plastic finite element analyses have been carried out for the evaluation of crack propagations in prestressed wires. The stress and stress intensity factors are calculated using virtual crack extension method and crack tip elements. The analysis results have been compared with available test results, and show at least $6 \%$ improved results from the linearly assumed analytical equations suggested by Forman et al. ${ }^{11)}$ In all, the current comparative work is not as conclusive as the nonlinear analysis. However, the previous analytical equation is economical but provides less information for the fracture of the section, which is affected by hydrogen induced cracking. To get over this, the present bi-linear elastic-plastic fracture analysis can be an alternative method for the improved estimation for the cracked section. The efficiency and additional improvements are likely to be increased as the more available test data obtained.
(3) At each time step, the crack propagates when the diffused hydrogen gas pressure creates critical stress intensity factors. A transient finite element program for the hydrogen diffusion analyses has been developed, which could be used for the parametric analysis and design of prestressed structures. For an example case, varying prestressing force effects to the hydrogen induced cracking problem in a prestressed concrete bridge, is discussed in a separate study.

\section{Acknowledgement}

This research was supported by the research grant from MOCT (Ministry of Construction and Technology) of Korean Government (Grant number: 06HIGH-TECH FUSION-E01) and Sustainable Building Research Center of Hanyang University which was supported the SRC/ ERC program of MOST (grant R11-2005-056-01003). The authors hereby express their sincere appreciation.

\section{REFERENCES}

1) FHWA, The Role of Smart Structures in Managing Aging Highway Infrastructure, http://www.tfhrc.gov/hnr20/nde/ppt/sld037.htm, United States Department of Transportation-Federal Highway Administration, (2000). (accessed on Aug. 5th, 2007)

2) H. Wang and A. A. Sagues: Corrosion of Post-Tensioning Strands, Florida Department of Transportation, Report BC353-33, (2005), 34.

3) G. H. Koch: Stress-Corrosion Cracking and Hydrogen Embrittlement, ASM Handbook, Vol. 19: Fatigue and Fracture, ASM Int., Virginia Tech. Printing Department, Blacksburg, VA, USA, (1996), 485.

4) A. H. M. Krom, R. W. J. Kores and A. Baker: J. Mech. Phys. Solids, 
ISIJ International, Vol. 48 (2008), No. 4

47 (1999), 971.

5) W. T. Tsai and S. L. Chou: Corros. Sci., 42 (2000), 1741.

6) H. P. V. Leeuwen: Eng. Fract. Mech., 6 (1974), 141.

7) A. T. Yokobori, Y. Chinda, T. Nemoto, K. Satoh and T. Yamada: Eng. Fract. Mech., 55 (1996), 47.

8) J. Lufrano, P. Sofronis and H. K. Birnbaum: J. Mech. Phys. Solids, 44 (1996), 179

9) J. Toribio: Corros. Sci., 39 (1997), 1687.

10) R. M. Mayrbaurl and S. Camo: J. Bridge Eng., 6 (2001), 645.

11) R. G. Forman and V. Shivakumar: Fract. Mech., 17 (1986), ASTM Special Technical Publication 905.

12) J. Toribio, B. González and J. C. Matos: Mater. Sci. Eng. A,
A468-470 (2007), 267.

13) F. P. Yang, Z. B. Kuang and V. N. Shlyannikov: Int. J. Fatigue, 28 (2006), 431.

14) H. D. Bui: Mecanique de laRupture Fragule, Mason Paris, (1978).

15) R. J. Hartranft and G. C. Sih: Eng. Fract. Mech., 9 (1977), 705.

16) X. B. Lin and R. A. Smith: Int. J. Fatigue, 19 (1997), 461.

17) R. S. Barsoum: Int. J. Number Meth. Eng., 10 (1976), 25.

18) B. Moran and C. F. Shih: Eng. Fract. Mech., 27 (1987), 615.

19) L. J. Segerlind: Applied Finite Element Analysis, Wiley Text Books, 2nd ed., (1984).

20) T. Cho: Doctoral Dissertation, The University of Michigan, (2003). 